\title{
Role the awareness of healthy nutrition and lifestyle for improving dietary behavior and the bodily status of employees agri-food industry
}

\author{
Abdelfettah Derouiche $^{1}$, Houria Makhlouki $^{1}$, Maria Elrbaoui ${ }^{1}$, Younes Elkardi ${ }^{1}$, Ali Jafri ${ }^{2}$ and \\ Chloé Martin ${ }^{3}$ \\ ${ }^{1}$ Human Nutrition Research Group Laboratory Biology and Health URAC34, FSBM Hassan II, Casablanca, Morocco, \\ ${ }^{2}$ Faculty of Health Sciences and Techniques, Mohammed VI University of Health Sciences, Casablanca, Morocco and \\ ${ }^{3}$ Danone Institute, Casablanca, Morocco
}

\section{Abstract}

Introduction: Awareness and education Nutritional contribute to the voluntary improvement of dietary behaviour and adopting healthy lifestyle. In a work environment, nutrition education falls under the social responsibility of the employer to promote healthier dietary choices. This study aims to assess the impact of nutrition education on dietary behaviour, lifestyle and body composition on a group of industry workers.

Materials and Methods: A group of 44 workers have volunteered to participate to this 6 months' trial. Participants were aged between 28 and 58 years old (43\% women and $57 \%$ men). All participants have completed a cap-score questionnaire at the beginning and end of the study, the questionnaire was organised into 3 sections: food groups, dietary behaviour, and lifestyle. Anthropometric measurements (height, weight, BMI, muscle mass, fat mass, hip circumference and waist circumference) were monitored during the study. Nutrition education sessions addressed 4 topics including healthy lifestyle. Participants were free to attend to any of the workshops.

Results: Only fifteen participants attended all sessions. Data showed that obesity lowered by $28.6 \%$ and overweight increased by $32.6 \%$, body composition changes were not significant. Changes in dietary behaviour were not significant, while scores of food groups and lifestyle changed significantly $(\mathrm{p}=0.053$ and $\mathrm{p}=0.042$ respectively).

Discussion: This study reveals the commitment of workers to nutrition education which could be a part in improving their dietary choices, their health and their productivity.

\section{Conflict of Interest}

There is no conflict of interest 